Article

\title{
Detecting Schisandrae Chinensis Fructus and Its Chinese Patent Medicines with a Nucleotide Signature
}

\author{
Wenjun Jiang ${ }^{1}$, Li Ren ${ }^{1}$, Mengyue Guo ${ }^{1}{ }^{\mathbb{D}}$, Nitin Mantri ${ }^{2}$, Sha Zhao ${ }^{1}$ and Xiaohui Pang ${ }^{1, *}$ \\ 1 Key Lab of Chinese Medicine Resources Conservation, State Administration of Traditional Chinese Medicine \\ of the People's Republic of China, Institute of Medicinal Plant Development, Chinese Academy of Medical \\ Sciences \& Peking Union Medical College, Beijing 100193, China; wenjunjiang0927@gmail.com (W.J.); \\ renliwangyi@hotmail.com (L.R.); guomy0908@hotmail.com (M.G.); zhaosha919@hotmail.com (S.Z.) \\ 2 The Pangenomics Group, School of Science, RMIT University, Melbourne 3083, Australia; \\ nitin.mantri@rmit.edu.au \\ * Correspondence: xhpang@implad.ac.cn; Tel.: +86-10-57833051
}

Received: 11 April 2019; Accepted: 17 May 2019; Published: 22 May 2019

\begin{abstract}
Schisandrae Chinensis Fructus (Wuweizi) is often adulterated with Schisandrae Sphenantherae Fructus (Nanwuweizi) in the herbal market. This adulteration is a threat to clinical treatment and safety. In this study, we aimed to develop a nucleotide signature for the identification of Wuweizi and its Chinese patent medicines based on the mini-DNA barcoding technique. We collected 49 samples to obtain internal transcribed spacer 2 (ITS2) sequences and developed a 26-bp nucleotide signature (5'-CGCTTTGCGACGCTCCCCTCCCTCCC-3') on the basis of a single nucleotide polymorphism (SNP) site within the ITS2 region that is unique to Wuweizi. Then, using the nucleotide signature, we investigated 27 batches of commercial crude drug samples labeled as Wuweizi and eight batches of Chinese patent medicines containing Wuweizi. Results showed that eight commercial crude drug samples were adulterants and one of the Chinese patent medicines contained adulterants. The nucleotide signature can serve as an effective tool for identifying Wuweizi and its Chinese patent medicines and can thus be used to ensure clinical drug safety.
\end{abstract}

Keywords: Wuweizi; Chinese patent medicines; identification; nucleotide signature

\section{Introduction}

Schisandra of the family Magnoliaceae is a genus with about 30 species, two of which, namely Schisandra chinensis (Turcz.) Baill. and Schisandra sphenanthera Rehd.et Wils., have a long history of clinical use in China. Schisandrae Chinensis Fructus, the dry ripe fruits of S. chinensis, is recorded as Wuweizi in Chinese pharmacopeia. It has functions of astringing and securing, tonifying qi and engendering fluid, as well as tonifying the kidney and calming the heart [1]. As a well-known traditional Chinese medicine, it has been used for thousands of years because of its outstanding physiological as well as therapeutic effects [2]. It has also been recognized as an official medicine in the Russian Pharmacopoeia since the early 1960s [3]. Modern pharmacological research demonstrates that the fruits of $S$. chinensis contain various active components, which act as hepatoprotective $[4,5]$, antioxidative [6], anti-inflammatory $[7,8]$, and anti-cancer agents $[9,10]$. Schisandrae Sphenantherae Fructus, the dry ripe fruits of S. sphenanthera, is listed in Chinese pharmacopeia as Nanwuweizi, but has been used as Wuweizi for a long time. Nanwuweizi is inferior to Wuweizi according to the ancient pharmaceutical book Shen Nong Ben Tsao Ching and this fact has been confirmed by several clinical studies [2,11]. Nanwuweizi was listed as a new entry in Chinese pharmacopeia in 2010 because its active components, medicinal values, and qualities are different from Wuweizi [12]. However, Wuweizi 
is often adulterated by Nanwuweizi in the herbal market because of their similar fruit morphologies (Figure 1) and the lower price of Nanwuweizi. To guarantee their safety and efficacy, the correct identification of Wuweizi and its Chinese patent medicines is highly desired.

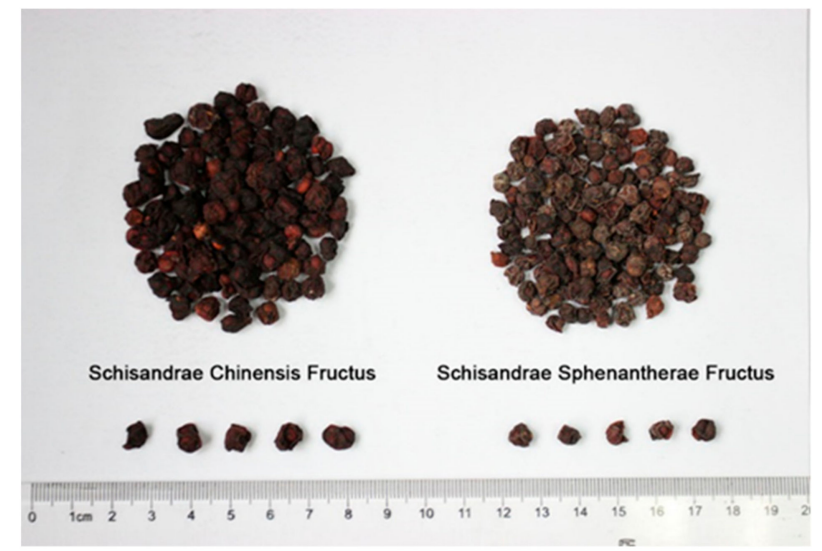

Figure 1. Samples of Schisandrae Chinensis Fructus and Schisandrae Sphenantherae Fructus collected from herbal market.

Distinguishing Wuweizi from Nanwuweizi through conventional taxonomic methods based on morphology is difficult because of their similar morphologies and more so when they are made into Chinese patent medicines. Previous studies reported that chemical analysis based on chromatographic techniques using schisandrin and deoxyschizandrin as markers can be used for the authentication of Wuweizi and Nanwuweizi. Such methods include thin layer chromatography, high performance liquid chromatography, and gas chromatography [13-15]. With the development of molecular biology techniques, polymerase chain reaction (PCR) based on molecular markers has been extensively applied in the identification of $S$. chinensis and S. sphenanthera. Sun et al. successfully used inter simple sequence repeat (ISSR) molecular markers to analyze population genetic variation between $S$. chinensis and S. sphenanthera [16]. Lee et al. demonstrated that Random amplifed polymorphic DNA (RAPD) -derived Sequence Characterized Amplified Region (SCAR) markers are useful in authenticating S. chinensis from other closely related species [17]. DNA barcoding has attracted considerable attention since Paul Hebert first proposed that cytochrome c oxidase 1 (CO1) can serve as a taxonomic tool for biological identification in 2003 [18]. At present, DNA barcoding has been extensively applied in the discrimination of medicinal plants and herbal materials, especially the internal transcribed spacer 2 (ITS2) barcode. Zhao et al. accurately distinguished Acanthopanacis cortex and its adulterants by using ITS2, indicating that ITS2 has great potential to be used for the supervision of the herbal market [19]. Guo et al. used ITS2 to authenticate medicinal plants and commercial samples in Cynanchum and showed that ITS2 has a high identification capability [20]. Li et al. successfully identified leaves of S. chinensis and S. sphenanthera using ITS2 [21].

Although DNA barcodes are effective for identifying herbs, they are difficult to be amplified from some processed samples with highly degraded DNA [22]. For instance, Lo et al. successfully amplified 88-bp DNA fragments from P. ginseng samples boiled for $120 \mathrm{~min}$ but failed to amplify longer fragments [23]. The reason for failure of amplifying longer fragments may be that the DNA template may have been too damaged to serve as a PCR template. Yuan et al. found that the efficiency of PCR amplification of $A$. dahurica decoction pieces decreases after baking at $80{ }^{\circ} \mathrm{C}$ [24]. The study demonstrated that short DNA sequences have advantages over long ones in terms of enhancing the success rate of amplification from processed samples. Given the difficulty the amplification of highly degraded samples poses, Meusnier et al. established a novel approach based on a DNA mini-barcode [25]. Their study indicated that the mini-barcode can be reliably obtained from archival specimens and dramatically broadens the applications of DNA barcoding. Little et al. designed a novel 
DNA mini-barcode and successfully applied it to the authentication of Ginkgo biloba in herbal dietary supplements [26]. Sarkinen et al. concluded that PCR success rate is strongly correlated with amplicon size; that is, the shorter fragments are more likely to achieve success [27]. The nucleotide signature, a fragment of one or more nucleotides unique to one species [28], can be used for rapidly and accurately authenticating samples with degraded DNA. Ng et al. successfully developed a nucleotide signature consisting of nine bases in the ITS region for the identification of Aglaia stellatopilosa [29]. Liu et al. successfully found a 27-bp nucleotide signature of American ginseng derived from the ITS2 region and used it to detect Chinese patent medicines [30]. Gao et al. successfully developed a 34-bp nucleotide signature for investigating the extracts and Chinese patent medicines containing Lonicerae japonicae Flos (Jinyinhua) and found many adulterated products [31]. Wang et al. successfully employed a 37-bp nucleotide signature to detect commercial products containing Angelicae sinensis radix (Danggui) and demonstrated that it can serve as a "species-specific marker" for Danggui [32].

To correctly identify Wuweizi and its Chinese patent medicines, the present study was designed to develop a Wuweizi-specific nucleotide signature. Finally, a 26-bp nucleotide signature (5'-CGCTTTGCGACGCTCCCCTCCCTCCC-3'), which is highly conserved and unique to S. chinensis, was found. To the best of our knowledge, this study is the first to use a nucleotide signature to identify Wuweizi and its Chinese patent medicines. The method based on the nucleotide signature will play a positive role in authenticating herbal materials and their products.

\section{Materials and Methods}

\subsection{Sampling of the Materials}

A total of 49 samples were collected from production areas, herbal markets, and drug stores of 14 provinces from China and Japan, including S. chinensis (27 fruits and one leaf sample) and S. sphenantherae (13 fruits, seven leaves and one seedling sample). These samples were identified by experts and voucher specimens were taken. All the corresponding voucher samples used in this study were deposited in the Herbarium of the Institute of Medicinal Plant Development, Chinese Academy of Medical Sciences, Beijing, China. ITS2 sequences were obtained and the nucleotide signature of S. chinensis was developed by using the above samples. The details of the reference samples for development of the nucleotide signature are listed in Table S1. A total of 143 ITS2 sequences from 18 Schisandra species were downloaded from GenBank and used to validate the nucleotide signature (Table S2). Then, 27 batches of commercial crude drug samples labeled as Wuweizi were gathered from ten different provinces of China and their authenticity were investigated (Table 1). Eight batches of Chinese patent medicines containing Wuweizi were purchased from Beijing and online drug stores to test if they contain adulterants (Table 2).

Table 1. Characteristics and identification results of commercial crude drug samples labeled as Schisandrae Chinensis Fructus.

\begin{tabular}{ccc}
\hline Voucher No. & Collection Location & Genuine \\
\hline DL01 & Dalian, Liaoning & Yes \\
DL02 & Dalian, Liaoning & Yes \\
BJ01 & Beijing & No \\
BJ02 & Beijing & Yes \\
XA01 & Xian, Shanxi & Yes \\
XA02 & Xian, Shanxi & Yes \\
WN01 & Weinan, Shanxi & No \\
NJ01 & Nanjing, Jiangsu & No \\
CZ01 & Changzhi, Shanxi & Yes \\
CZ02 & Changzhi, Shanxi & No \\
CZ03 & Changzhi, Shanxi & Yes \\
CZ04 & Changzhi, Shanxi & No \\
CZ05 & Changzhi, Shanxi & No \\
\hline
\end{tabular}


Table 1. Cont.

\begin{tabular}{ccc}
\hline Voucher No. & Collection Location & Genuine \\
\hline CZ06 & Changzhi, Shanxi & No \\
CZ07 & $\begin{array}{c}\text { Changzhi, Shanxi } \\
\text { online store } \\
\text { (Aomiao) }\end{array}$ & No \\
OL01 & online store & Yes \\
& (Caobenyunnan) & Yes \\
OL02 & Chengdu, Sichuan & Yes \\
CD01 & Chengdu, Sichuan & Yes \\
CD02 & Bozhou, Anhui & Yes \\
BZ01 & Bozhou, Anhui & Yes \\
BZ02 & Bozhou, Anhui & Yes \\
BZ03 & Bozhou, Anhui & Yes \\
BZ04 & Anguo, Hebei & Yes \\
AG01 & Tonghua, Jilin & Yes \\
TH01 & Tonghua, Jilin & Yes \\
TH02 & Lincun, Jilin & Yes \\
LC01 & & \\
& & \\
\hline
\end{tabular}

Table 2. Characteristics of eight Chinese patent medicines.

\begin{tabular}{|c|c|c|c|c|}
\hline $\begin{array}{l}\text { Voucher } \\
\text { No. }\end{array}$ & Samples & Listed Ingredients on Label & $\begin{array}{l}\text { Collection } \\
\text { Location }\end{array}$ & Genuine \\
\hline TW01 & $\begin{array}{l}\text { Tianwang Buxin } \\
\text { Pills }\end{array}$ & $\begin{array}{l}\text { Schisandrae Chinensis Fructus, Salviae } \\
\text { Miltiorrhizae Radix Et Rhizoma, Angelicae } \\
\text { Sinensis Radix, Acori Tatarinowii Rhizoma, } \\
\text { Codonopsis Radix, Poria, Ophiopogonis Radix, } \\
\text { Asparagi Radix, Rehmanniae Radix, } \\
\text { Scrophulariae Radix, Polygalae Radix, Ziziphi } \\
\text { Spinosae Semen, Platycladi Semen, Platycodonis } \\
\text { Radix, Glycyrrhizae Radix Et Rhizoma, } \\
\text { Cinnabaris }\end{array}$ & Beijing store & Yes \\
\hline TW02 & $\begin{array}{l}\text { Tianwang Buxin } \\
\text { Pills }\end{array}$ & $\begin{array}{l}\text { Schisandrae Chinensis Fructus, Salviae } \\
\text { Miltiorrhizae Radix Et Rhizoma, Angelicae } \\
\text { Sinensis Radix, Acori Tatarinowii Rhizoma, } \\
\text { Codonopsis Radix, Poria, Ophiopogonis Radix, } \\
\text { Asparagi Radix, Rehmanniae Radix, } \\
\text { Scrophulariae Radix, Polygalae Radix, Ziziphi } \\
\text { Spinosae Semen, Platycladi Semen, Platycodonis } \\
\text { Radix, Glycyrrhizae Radix Et Rhizoma, } \\
\text { Cinnabaris }\end{array}$ & online shop & Yes \\
\hline MW01 & $\begin{array}{l}\text { Maiwei Dihuang } \\
\text { Pills }\end{array}$ & $\begin{array}{l}\text { Schisandrae Chinensis Fructus, Ophiopogonis } \\
\text { Radix, Rehmanniae Radix Praeparata, Corni } \\
\text { fructus, Moutan Cortex, Dioscoreae Rhizoma, } \\
\text { Poria, Alismatis Rhizoma }\end{array}$ & Beijing store & Yes \\
\hline MW02 & $\begin{array}{l}\text { Maiwei Dihuang } \\
\text { Pills }\end{array}$ & $\begin{array}{l}\text { Schisandrae Chinensis Fructus, Ophiopogonis } \\
\text { Radix, Rehmanniae Radix Praeparata, Corni } \\
\text { fructus, Moutan Cortex, Dioscoreae Rhizoma, } \\
\text { Poria, Alismatis Rhizoma }\end{array}$ & online shop & Yes \\
\hline BT & Bantu Pills & $\begin{array}{l}\text { Schisandrae Chinensis Fructus, Rehmanniae } \\
\text { Radix, Rehmanniae Radix Praeparata, Polygoni } \\
\text { Multiflori Radix Praeparata, Angelicae Sinensis } \\
\text { Radix, Salviae Miltiorrhizae Radix Et Rhizoma, } \\
\text { Paeoniae Radix Alba, Notopterygii Rhizoma Et } \\
\text { Radix, Chaenomelis Fructus }\end{array}$ & online shop & Yes \\
\hline
\end{tabular}


Table 2. Cont

\begin{tabular}{|c|c|c|c|c|}
\hline $\begin{array}{l}\text { Voucher } \\
\text { No. }\end{array}$ & Samples & Listed Ingredients on Label & $\begin{array}{l}\text { Collection } \\
\text { Location }\end{array}$ & Genuine \\
\hline $\mathrm{SH}$ & $\begin{array}{l}\text { Shihu Yeguang } \\
\text { Pills }\end{array}$ & $\begin{array}{l}\text { Schisandrae Chinensis Fructus, Dendrobii Caulis, } \\
\text { Ginseng Radix Et Rhizoma, Dioscoreae Rhizoma, } \\
\text { Poria, Glycyrrhizae Radix Et Rhizoma, } \\
\text { Cistanches Herba, Lycii Fructus, Cuscutae Semen, } \\
\text { Rehmanniae Radix, Rehmanniae Radix } \\
\text { Praeparata, Asparagi Radix, Ophiopogonis Radix, } \\
\text { Armeniacae Semen Amarum, Saposhnikoviae } \\
\text { Radix, Chuanxiong Rhizoma, Aurantii Fructus, } \\
\text { Coptidis Rhizoma, Achyranthis Bidentatae Radix, } \\
\text { Chrysanthemi Flos, Tribuli Fructus, Celosiae } \\
\text { Semen, Cassiae Semen, Bubali Cornu, Saigae } \\
\text { Tataricae Cornu }\end{array}$ & online shop & Yes \\
\hline AS & Anshen Capsules & $\begin{array}{l}\text { Schisandrae Chinensis Fructus, } \\
\text { Ziziphispinosaesemen, Chuanxiong Rhizoma, } \\
\text { Anemarrhenae Rhizoma, Ophiopogonis Radix, } \\
\text { Polygoni Multiflori Radix Praeparata, Salviae } \\
\text { Miltiorrhizae Radix Et Rhizoma, Poria }\end{array}$ & online shop & Yes \\
\hline
\end{tabular}

\subsection{DNA Extraction, PCR Amplification, and Sequencing}

\subsubsection{Fruit, Leaf, and Seedling}

The surface of materials containing fruits and leaves were wiped with $75 \%$ ethanol for sterilization and subsequently ground into powder with FastPrepbead mill (Retsch MM400, Haan, Germany) at a frequency of $30 \mathrm{~Hz}$. Genomic DNA was extracted from about $20 \mathrm{mg}$ of leaf powder and $40 \mathrm{mg}$ of fruit powder using the Plant Universal Genomic DNA Kit (Tiangen Biotech Co., Beijing, China) following the manufacturer's instructions. Polymerase chain reaction (PCR) amplification and sequencing were performed according to previous research [33].

\subsubsection{Commercial Crude Drug Sample and Chinese Patent Medicine}

We randomly selected one sample (about $40 \mathrm{mg}$ ) in each batch of commercial crude drugs and 1-3 samples (200-300 mg) in each batch of Chinese patent medicines. The samples were ground into powder using a FastPrep bead mill (Retsch MM400). DNA was extracted from powder using the Plant Universal Genomic DNA Kit and amplified by ITS2F. (5'-ATGCGATACTTGGTGTGAAT-3') /WWZ5R (5'-GCTCCTCGCAAACACCATAC-3') primer pairs that were newly designed by Primer 6.0 software (Premier Biosoft International, Palo Alto, CA, USA) and specific for S. chinensis and its closely related species. The location of primer pairs is shown in Figure 2. PCR was performed in a $25 \mu \mathrm{L}$ reaction system containing $2 \mu \mathrm{L}$ (about $30 \mathrm{ng}$ ) of DNA templates, $1.0 \mu \mathrm{L}$ of primer ITS2F/WWZ5R $(2.5 \mu \mathrm{mol} / \mathrm{L}), 12.5 \mu \mathrm{L}$ of 2x PCR Master Mix (Aidlab Biotechnologies Co., Ltd., Beijing, China), and double-distilled water. The reactions were performed with the same thermal program that was used for the other materials. Purified PCR products were sequenced in both directions with the newly designed primer pairs by the Sanger sequencing method on a $3730 \mathrm{XL}$ sequencer (Applied Biosystems, Inc., Foster City, CA, USA).

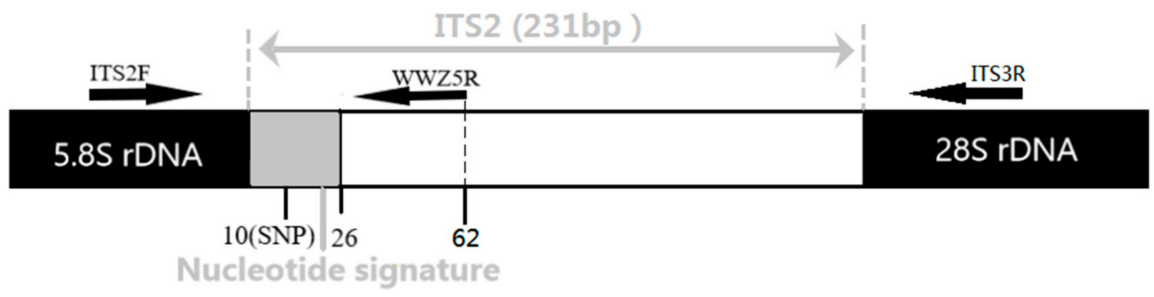

Figure 2. Schematic diagram showing the location of primer pairs (ITS2F/WWZ5R, ITS2F/ITS3R) and the nucleotide signature region of $S$. chinensis. 


\subsection{Sequence Analysis}

Raw ITS2 trace files generated from our study were assembled using CodonCode Aligner V.8.01 (CodonCode Co., Dedham, MA, USA). All the ITS2 sequences of Schisandra species, also including those downloaded from GenBank, were annotated as well as trimmed through a hidden Markov Model-based method to obtain the complete ITS2 regions [34]. The haplotypes of Schisandra species were selected by CodonCode Aligner and then aligned with MEGA 7.0 software [35]. The unique region was finally verified by BLAST analysis.

\section{Results}

\subsection{Development of Nucleotide Signature for S. chinensis}

All materials of fruits as well as leaves were successfully amplified using the primer pair ITS $2 \mathrm{~F} / 3 \mathrm{R}$ and sequenced. A total of 49 ITS2 sequences of S. chinensis and S. sphenanthera produced by experimental materials were aligned. The results of the alignment demonstrated that the intra-specific genetic divergences of $S$. chinensis and S. sphenanthera were low and the nucleotide (A) at position 10 was unique to $S$. chinensis. On the basis of the special SNP site, we successfully developed a 26-bp special nucleotide signature (5'-CGCTTTGCGACGCTCCCCTCCCTCCC-3') for the identification of S. chinensis.

\subsection{Evaluation of the Nucleotide Signature}

For the evaluation of the uniqueness and specificity of the 26-bp nucleotide signature, the 18 haplotypes of the 143 ITS2 sequences representing 18 Schisandra species were aligned using MEGA 7 (Figure 3) [35]. The result of the alignment confirmed the presence of one or two divergent nucleotides within the 26-bp special nucleotide signature between S. chinensis and other Schisandra species. In addition, the BLAST was conducted on GenBank using the 26-bp special nucleotide signature of $S$. chinensis as the query sequence. The results showed that the identification score of S. chinensis is $100 \%$, while for other species, even for closely related species, the identification score was less than $100 \%$ (Table 3). Therefore, the 26-bp nucleotide signature, newly developed in the present study, can serve as a unique tool for distinguishing $S$. chinensis from the other species presented in GenBank.
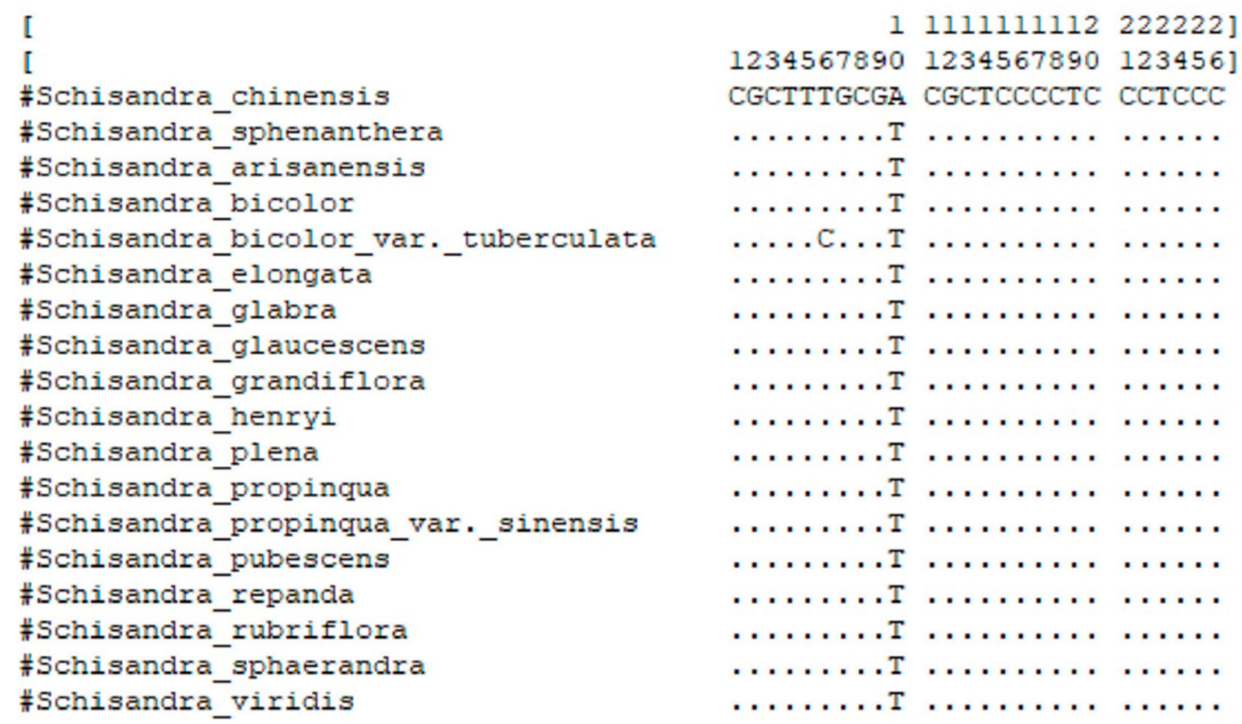

Figure 3. Alignment of the 26-bp nucleotide conserved region. 
Table 3. BLAST analysis of the 26-bp unique region.

\begin{tabular}{|c|c|c|c|c|c|c|c|}
\hline $\begin{array}{c}\text { Source Species of } \\
\text { Nucleotide } \\
\text { Signature }\end{array}$ & $\begin{array}{l}\text { Blast Result } \\
\text { in NCBI }\end{array}$ & $\begin{array}{l}\text { Number of } \\
\text { the Species }\end{array}$ & Max Score & $\begin{array}{l}\text { Total } \\
\text { Score }\end{array}$ & $\begin{array}{l}\text { Query } \\
\text { Cover }\end{array}$ & E Value & $\begin{array}{l}\text { Per. } \\
\text { Ident }\end{array}$ \\
\hline \multirow{11}{*}{ Schisandra chinensis } & Schisandra chinensis & 39 & 52.0 & 52.0 & $100 \%$ & 0.0003 & $100 \%$ \\
\hline & Schisandra sphenanthera & 1 & 44.1 & 44.1 & $100 \%$ & 0.065 & $96.15 \%$ \\
\hline & Kadsura heteroclite & 1 & 44.1 & 44.1 & $100 \%$ & 0.065 & $96.15 \%$ \\
\hline & Kadsura interior & 13 & 44.1 & 44.1 & $100 \%$ & 0.065 & $96.15 \%$ \\
\hline & Schisandra sphaerandra & 2 & 44.1 & 44.1 & $100 \%$ & 0.065 & $96.15 \%$ \\
\hline & Schisandra henryi & 2 & 44.1 & 44.1 & $100 \%$ & 0.065 & $96.15 \%$ \\
\hline & Schisandra rubriflora & 4 & 44.1 & 44.1 & $100 \%$ & 0.065 & $96.15 \%$ \\
\hline & Schisandra propinqua & 2 & 44.1 & 44.1 & $100 \%$ & 0.065 & $96.15 \%$ \\
\hline & Schisandra henryi & 3 & 44.1 & 44.1 & $100 \%$ & 0.065 & $96.15 \%$ \\
\hline & Kadsura longipedunculata & 27 & 44.1 & 44.1 & $100 \%$ & 0.065 & $96.15 \%$ \\
\hline & Kadsura heteroclita & 6 & 44.1 & 44.1 & $100 \%$ & 0.065 & $96.15 \%$ \\
\hline
\end{tabular}

\subsection{Identification of Commercial Samples}

\subsubsection{Commercial Crude Drug Samples}

A total of 27 batches of commercial crude drugs labeled as Wuweizi were purchased from drug stores, herbal markets, and online stores. All the tested samples of commercial crude drugs were successfully amplified (170 bp) with the primer pair ITS2F/WWZ5R and then bidirectionally sequenced. Of the 27 samples tested, 19 were authenticated as Wuweizi and eight were adulterants. The identification results and other data of the samples investigated in our study are provided in Table 1.

\subsubsection{Chinese Patent Medicines}

Eight batches of Chinese patent medicines containing Wuweizi were tested by the primer pair ITS2F/WWZ5R, including Tianwang Buxin Pills, Zengguang Tablets, Maiwei Dihuang Pills, Shihu Yeguang Pills, Bantu Pills, and Anshen Capsules. The details of the Chinese patent medicines and identification results are provided in Table 2. The targeted nucleotide signature of $S$. chinensis was successfully amplified (170 bp) and sequenced from these batches. After analyzing the nucleotide signature and double peaks at the SNP position (Figure 4), we found that one batch (Zengguang Tablets) contained adulterants and seven were authentic. 


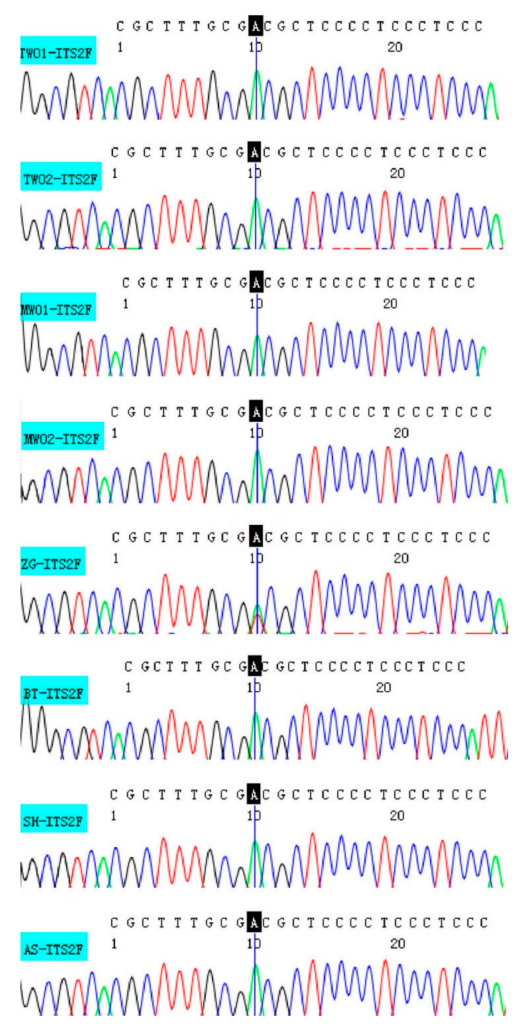

Figure 4. Single nucleotide polymorphisms (SNP) peaks of four batches of Chinese patent medicine containing Wuweizi.

\section{Discussion}

\subsection{The Necessity of Developing Nucleotide Signature for Chinese Patent Medicines}

S. chinensis is widely distributed in several provinces in northeastern China, eastern Russia, Japan, and Korea [36]. This species is not only a medicinal material recorded in Chinese Pharmacopeia and Russian Pharmacopoeia, but also an important raw material for various Chinese patent medicines, such as Tianwang Buxin Pills, Zengguang Tablets and Anshen Capsules. The contents of schisandrin and schisantherin A are used as the quality control indicators for Wuweizi and Nanwuweizi, respectively, in the Chinese Pharmacopoeia (2015 edition) [1]. However, Chinese patent medicines are composed of a considerable number of herbs and each one may contain dozens or even hundreds of chemical ingredients. Adulterants may occur considering the similar chemical constituents in herbal medicines from the same genus, which is a threat to drug safety and the health of consumers. Thus, it is not sufficient to identify the composition of Chinese patent medicines by content determination alone. Developing a novel approach for differentiating ingredients listed on the label is therefore highly desirable and is an initial step for ensuring the efficacy of Chinese patent medicines.

In previous research, Li et al. used ITS2 to distinguish S. chinensis from S. sphenanthera and found that ITS2 is useful for the authentication of S. chinensis [21]. All the materials they used were leaves, so it is not known whether ITS2 is suitable for identifying other materials of $S$. chinensis, such as herbal materials and Chinese patent medicines. Considering that the mini-DNA barcoding technique is more suitable for the identification of processed samples with heavy DNA degradation, it was used in our study. We found a 26-bp nucleotide signature unique to $S$. chinensis for the first time, which can be used to identify S. chinensis, including plants, crude drugs, and Chinese patent medicines. 


\subsection{Nucleotide Signature Can Effectively Detect Wuweizi and Its Chinese Patent Medicines}

A total of 27 batches of commercial crude drug samples and eight batches of Chinese patent medicines were surveyed in this study. The nucleotide signature was used in the authentication of the tested samples and it was confirmed to be efficient in identifying Wuweizi. First, the newly developed nucleotide signature was verified by BLAST analysis, which displayed perfect identification capability with 100\% identification efficiency at the species level. Second, presence of adulterants in the Chinese patent medicines was determined through SNP method. Chen et al. found that a mixed powder of American ginseng and Asian ginseng can be clearly detected by analyzing the double peaks at the SNP site even when the mixing ratio was as low as $5 \%$ [37]. Similarly, in our study, S. chinensis and other species was efficiently distinguished by analyzing the double peaks at the SNP site.

\subsection{Nucleotide Signature Is a Powerful Technique for Herbal Market Supervision}

The DNA barcoding technique has been used for investigating the commercial products in the herbal market recently. For instance, Ruhsam et al. used DNA barcoding to investigate Eleutherococcus and Rhodiola herbal supplements and found that the phenomenon of substitution or adulteration is common [38]. Han et al. utilized DNA barcoding to survey 295 herbal materials and found that approximately $4.2 \%$ of the samples are adulterants [39]. The studies revealed that DNA barcodes are useful in identifying some commercial herbs. However, it is difficult to successfully amplify DNA barcodes for Chinese patent medicines with severely degraded DNA. The nucleotide signature is a species-specific marker much shorter than DNA barcodes, which gives it an advantage in detecting the ingredients of Chinese patent medicines. In our study, the newly identified 26-bp nucleotide signature was successfully used for the authentication of Wuweizi and its Chinese patent medicines. As shown in Table 1, eight of 27 of the tested commercial crude samples were adulterants. Meanwhile, one of the eight batches of Chinese patent medicines contained adulterants. Thus, the nucleotide signature is an efficient tool for identifying samples with heavy DNA degradation, providing a useful tool for herbal market supervision.

\section{Conclusions}

Our study revealed that the adulteration of Wuweizi is not uncommon in the herbal market, emphasizing the need for market supervision. The 26-bp nucleotide signature we developed in this study can serve as a unique tool for identifying and detecting Wuweizi and its Chinese patent medicines. The nucleotide signature has potential to be used to supervise the herbal market, thereby ensuring the efficacy and safety of drugs.

Supplementary Materials: The following are available online at http://www.mdpi.com/2073-4425/10/5/397/s1, Table S1. Location of collection of reference samples for the development of the nucleotide signature. Table S2. Characteristics of the Schisandra genus derived from GenBank. Figure S1. Alignment of the ITS2 region of experimental materials.

Author Contributions: Conceptualization, X.P.; data curation, W.J., L.R. and M.G.; formal analysis, W.J. and X.P.; funding acquisition, X.P.; investigation, L.R.; methodology, W.J. and L.R.; resources, S.Z.; validation, X.P.; writing—original draft, W.J.; writing—review and editing, N.M. and X.P.

Funding: This work was supported by grants from the National Natural Science Foundation of China (No. 81573541) and the State Scholarship Fund of China (No. 201808110108) to XP.

Conflicts of Interest: The authors declare no conflict of interest.

\section{References}

1. Chinese Pharmacopoeia Commission. Pharmacopoeia of the People's Republic of China, 1st ed.; Press of Chinese Medical Science and Technology: Beijing, China, 2015; pp. 66-67 and 244.

2. Lu, Y.; Chen, D.-F. Analysis of Schisandra chinensis and Schisandra sphenanthera. J. Chromatogr. A. 2009, 1216, 1980-1990. [CrossRef] 
3. USSR Ministry of Health. The State Pharmacopoeia of the Union Soviet Socialist Republics, 10th ed.; USSR Ministry of Health: Moscow, Russia, 1966.

4. Zhu, M.; Lin, K.F.; Yeung, R.Y.; Li, R.C. Evaluation of the protective effects of Schisandra chinensis on Phase I drug metabolism using a CCl4 intoxication model. J. Ethnopharmacol. 1999, 67, 61-68. [CrossRef]

5. Pan, S.Y.; Dong, H.; Zhao, X.Y.; Xiang, C.J.; Fang, H.Y.; Fong, W.F.; Yu, Z.L.; Ko, K.M. Schisandrin B from Schisandra chinensis reduces hepatic lipid contents in hypercholesterolaemic mice. J. PharmPharmacol. 2008, 60, 399-403. [CrossRef]

6. Cheng, N.; Ren, N.; Gao, H.; Lei, X.; Zheng, J.; Cao, W. Antioxidant and hepatoprotective effects of Schisandra chinensis pollen extract on CCl4-induced acute liver damage in mice. Food Chem. Toxicol. 2013, 55, 234-240. [CrossRef] [PubMed]

7. Oh, S.Y.; Kim, Y.H.; Bae, D.S.; Um, B.H.; Pan, C.H.; Kim, C.Y.; Lee, H.J.; Lee, J.K. Anti-inflammatory effects of gomisin N, gomisin J, and schisandrin C isolated from the fruit of Schisandra chinensis. Biosci. Biotech. Bioch. 2010, 74, 285-291. [CrossRef]

8. Guo, L.Y.; Hung, T.M.; Bae, K.H.; Shin, E.M.; Zhou, H.Y.; Hong, Y.N.; Kang, S.S.; Kim, H.P.; Kim, Y.S. Anti-inflammatory effects of schisandrin isolated from the fruit of Schisandra chinensis Baill. Eur. J. Pharmacol. 2008, 591, 293-299. [CrossRef]

9. Hwang, D.; Shin, S.Y.; Lee, Y.; Hyun, J.; Yong, Y.; Park, J.C.; Lee, Y.H.; Lim, Y. A compound isolated from Schisandra chinensis induces apoptosis. Bioorg. Med. Chem. Lett. 2011, 21, 6054-6057. [CrossRef]

10. Kim, S.J.; Min, H.Y.; Lee, E.J.; Kim, Y.S.; Bae, K.; Kang, S.S.; Lee, S.K. Growth inhibition and cell cycle arrest in the G0/G1 by schizandrin, a dibenzocyclooctadiene lignan isolated from Schisandra chinensis, on T47D human breast cancer cells. Phytother. Res. 2010, 24, 193-197. [CrossRef] [PubMed]

11. Zhang, S.N; Wu, S.X. Advances in chemical and pharmacological effects of volatile oil from Schisandra fruits. J. Chin. Med. Mater. 2007, 30, 118-120.

12. Hu, Y.J.; Chen, J.Z.; Ye, L. The study evolvement of chemic components and diferentiation methods between Schisandra chinensis and Schisandra sphenanthera. Res. Pr. Chin. Med. 2008, 22, 59-62.

13. Jiang, P.; Lu, Y.; Chen, D. Authentication of Schisandra chinensis and Schisandra sphenanthera in Chinese patent medicines. J. Pharmaceut. Biomed. 2016, 131, 263-271. [CrossRef]

14. Zhu, M.; Cao, Y.; Fan, G. Microwave-Assisted Extraction and Fingerprint Studies of Schisandra Chinensis (Turcz.) by High Performance Liquid Chromatography. J. Liq. Chromatogr. R. T. 2007, 30, 123-133. [CrossRef]

15. Wei, H.; Sun, L.; Tai, Z.; Gao, S.; Xu, W.; Chen, W. A simple and sensitive HPLC method for the simultaneous determination of eight bioactive components and fingerprint analysis of Schisandra sphenanthera. Anal. Chim. Acta 2010, 662, 97-104. [CrossRef] [PubMed]

16. Sun, Y.; Wen, X.; Huang, H. Population genetic differentiation of Schisandra chinensis and Schisandra sphenanthera as revealed by ISSR analysis. Biochem. Syst. Ecol. 2010, 38, 257-263. [CrossRef]

17. Lee, Y.M.; Moon, B.C.; Ji, Y.; Seo, H.S.; Kim, H.K. Development of RAPD-Derived SCAR Markers and Multiplex-PCR for Authentication of the Schisandrae Fructus. Korean J. Med. Crop Sci. 2013, 21, 165-173. [CrossRef]

18. Hebert, P.D.; Cywinska, A.; Ball, S.L.; Dewaard, J.R. Biological identifications through DNA barcodes. P. Roy. Soc. B Biol. Sci. 2003, 270, 313-321. [CrossRef]

19. Zhao, S.; Chen, X.; Song, J.; Pang, X.; Chen, S. Internal transcribed spacer 2 barcode: A good tool for identifying Acanthopanacis cortex. Front. Plant Sci. 2015, 6, 840. [CrossRef]

20. Guo, M.; Ren, L.; Pang, X. Inspecting the True Identity of Herbal Materials from Cynanchum Using ITS2 Barcode. Front. Plant Sci. 2017, 8, 1945. [CrossRef] [PubMed]

21. Li, X.-k.; Wang, B.; Han, R.-C.; Zheng, Y.-C.; Yin, H.-b.Y.; Xu, L.; Zhang, J.-K.; Xu, B.-1. Identification of medicinal plant Schisandra chinensis using a potential DNA barcode ITS2. Acta Soc. Bot. Pol. 2013, 82, $283-288$. [CrossRef]

22. Little, D.P. A DNA mini-barcode for land plants. Mol. Ecol. Resour. 2014, 14, 437-446. [CrossRef] [PubMed]

23. Lo, Y.T.; Li, M.; Shaw, P.C. Identification of constituent herbs in ginseng decoctions by DNA markers. Chin. Med. 2015, 10, 1. [CrossRef]

24. Yuan, Q.J.; Zhang, B.; Jiang, D.; Zhang, W.J.; Lin, T.Y.; Wang, N.H.; Chiou, S.J.; Huang, L.Q. Identification of species and materia medica within Angelica L. (Umbelliferae) based on phylogeny inferred from DNA barcodes. Mol. Ecol. Resour. 2015, 15, 358-371. [CrossRef] 
25. Meusnier, I.; Singer, G.A.C.; Landry, J.-F.; Hickey, D.A.; Hebert, P.D.N.; Hajibabaei, M. A universal DNA mini-barcode for biodiversity analysis. BMC Genomics 2008, 9, 214. [CrossRef]

26. Little, D.P. Authentication of Ginkgo biloba herbal dietary supplements using DNA barcoding. Genome 2014, 57, 513-516. [CrossRef]

27. Särkinen, T.; Staats, M.; Richardson, J.E.; Cowan, R.S.; Bakker, F.T. How to open the treasure chest? Optimising DNA extraction from herbarium specimens. PloS ONE 2012, 7(8), e43808. [CrossRef]

28. Gibbs, A.J.; Armstrong, J.S.; Gibbs, M.J. A type of nucleotide motif that distinguishes tobamovirus species more efficiently than nucleotide signatures. Arch. Virol. 2003, 149, 1941-1954. [CrossRef]

29. Ng, B.L.N.; Omarzuki, M.; Lau, G.S.K.; Pannell, C.M.; Yeo, T.C. A Nucleotide Signature for Identification of Aglaia stellatopilosa Pannell. Mol. Biotechnol. 2014, 56, 671-679. [CrossRef]

30. Liu, Y.; Wang, X.; Wang, L.; Chen, X.; Pang, X.; Han, J. A Nucleotide Signature for the Identification of American Ginseng and Its Products. Front. Plant Sci. 2016, 7, 319. [CrossRef]

31. Gao, Z.; Liu, Y.; Wang, X.; Song, J.; Chen, S.; Ragupathy, S.; Han, J.; Newmaster, S.G. Derivative Technology of DNA Barcoding (Nucleotide Signature and SNP Double Peak Methods) Detects Adulterants and Substitution in Chinese Patent Medicines. Sci. Rep. 2017, 7, 5858. [CrossRef]

32. Wang, X.; Liu, Y.; Wang, L.; Han, J.; Chen, S. A Nucleotide Signature for the Identification of Angelicae Sinensis Radix (Danggui) and Its Products. Sci. Rep. 2016, 6, 34940. [CrossRef]

33. Chen, S.; Yao, H.; Han, J.; Liu, C.; Song, J.; Shi, L.; Zhu, Y.; Ma, X.; Gao, T.; Pang, X.; et al. Validation of the ITS2 region as a novel DNA barcode for identifying medicinal plant species. PloS ONE 2010, 5, e8613. [CrossRef] [PubMed]

34. Keller, A.; Schleicher, T.; Schultz, J.; Muller, T.; Dandekar, T.; Wolf, M. 5.8 S-28S rRNA interaction and HMM-based ITS2 annotation. Gene 2009, 430, 50-57. [CrossRef]

35. Tamura, K.; Peterson, D.; Peterson, N.; Stecher, G.; Nei, M.; Kumar, S. MEGA5: Molecular evolutionary genetics analysis using maximum likelihood, evolutionary distance, and maximum parsimony methods. Mol. Biol. Evol. 2011, 28, 2731-2739. [CrossRef]

36. Flora of China Editorial Committee. Flora of China; Press of Science and Missouri Botanical Garden: Beijing, China, 2008; Volume 7, pp. 41-47.

37. Chen, X.; Liao, B.; Song, J.; Pang, X.; Han, J.; Chen, S. A fast SNP identification and analysis of intraspecific variation in the medicinal Panax species based on DNA barcoding. Gene 2013, 530, 39-43. [CrossRef] [PubMed]

38. Ruhsam, M.; Hollingsworth, P.M. Authentication of eleutherococcus and rhodiola herbal supplement products in the United Kingdom. J. Pharmaceut. Biomed. 2018, 149, 403-409. [CrossRef] [PubMed]

39. Han, J.; Pang, X.; Liao, B.; Yao, H.; Song, J.; Chen, S. An authenticity survey of herbal medicines from markets in China using DNA barcoding. Sci. Rep. 2016, 6, 18723. [CrossRef] [PubMed] 